\title{
An Intentional Aconite Overdose: A Case Report
}

\author{
Christopher Wood ${ }^{1}$, James Coulson ${ }^{2}$, John Thompson ${ }^{2}$, Stephen Bonner ${ }^{1}$ \\ 1 James Cook University Hospital, Middlesbrough, United Kingdom \\ 2 National Poisons Information Service, United Kingdom
}

\begin{abstract}
Background: Aconite is one of the most toxic known herbs, widely used for centuries as an essential Chinese medicine, but also for deliberate poisoning throughout history. Clinically indicated in herbal medicine for a range of ailments from headaches to muscle spasm, unfortunately the narrow therapeutic window may lead to a range of toxic presentations. The mechanism of action of the pharmacologically active compounds in Aconite relate to the activation of voltage gated sodium channels within a range of tissue including myocardial, neuronal and smooth muscle leading to persistent cellular activity. Case presentation: We report on a rare case of a fifty year old male with intentional aconite overdose presenting with refractory cardiovascular instability from persistent life threatening arrhythmias, respiratory failure and seizure activity. Conclusion: An overview of Aconite, its history, pharmacological effects, treatment of overdose and outcomes is presented.
\end{abstract}

Keywords: Aconite, cardiovascular

Received: 23 February 2020 / Accepted: 22 March 2020

\section{BACKGROUND}

Aconitum is a genus of over two hundred species of flowering plants which contain active ingredients containing aconite and the related alkaloids, lycaconitine and napelline. Chinese herbalists have actively used aconite for centuries for the treatment of muscular-skeletal disorders due to its analgesic and anti-inflammatory properties. It is derived from the plant Aconitum napellus, also known as Monkshood or Wolfsbane. However, only a few cases have been described in western literature in the past twenty years, despite being one of the most toxic plants in the UK.

Presentation relates not only to the site of absorption of aconite but also plasma concentration of active alkaloids, which varies within the individual parts of plants such as their roots or leaves but also between plants and the processing techniques used during agriculture[1].

Aconite's pharmacokinetic data has been clarified by several studies [2,3]. Following enteral absorption, aconite mediates a range of cellular effects via voltagegated sodium channels. Poisoning, with myocardial complications, usually develops within 15-30 minutes following ingestion of the plant leaves or roots. The effects of poisoning are classically dose related [4]. Aco- nite alkaloids undergo elimination by first-order kinetics primarily via renal excretion, with a minor pathway involving cytochrome P450 (CYP 3A)[5]. Aconite's half-life $\left(t_{1 / 2}\right)$ exhibits significant inter-individual variability up to sixteen hours but also variation between other alkaloids within the same family [6].

The structure-activity relationship of aconite is yet to be fully elucidated. Classically, aconite binds avidly and induces direct confirmation change to open voltage-gated sodium channels by binding site two of the alpha-subunit, with increased preferential binding to highly active channels, which promotes persistent channel opening, enhancing intracellular $\left[\mathrm{Na}^{+}\right]$and $\left[\mathrm{Ca}^{2+}\right]$, maintaining membrane depolarisation [7]. Myocardial cells classically enhance automaticity, but such activity is also observed in neuronal tissue and finally striated and smooth muscles.

\section{CAse Presentation}

A fifty-year-old professionally educated Caucasian male was found collapsed within a public area. Local hospitality staff informed emergency services. A suicide note stating he had consumed a quantity of Wolfsbane root and alcohol, was found with him. Paramedics ar- 
rived within ten minutes. Their assessment identified a confused, intoxicated, cyanotic individual with sporadic episodes of rigidity, tachycardia (105 beats.mins ${ }^{-1}$ ), hypotension (97/63 $\mathrm{mmHg}$ ) and hyperventilation (45 breaths.mins ${ }^{-1}$ ), frothing at his mouth. An electrocardiogram (ECG) (Figure 1) was performed identifying an irregular rhythm with left bundle branch block, multifocal ectopic and first degree AV block. Supportive care was initiated, an 18 gauge cannula was inserted and fluid administrated (1000ml of Ringers Lactate Solution).

He was transferred to an emergency department of a large tertiary centre (England, UK). During the transfer journey he required intermittent assistant ventilation with a bag valve mask due to periods of apnoea. On arrival at the emergency department, he suffered a seizure, which spontaneously resolved in three minutes. A medical history was taken and the patient was examined revealing a Glasgow Coma Scale score of 12, with progressive respiratory failure and haemodynamic instability (BP $60 / 40 \mathrm{mmHg}$ ) secondary to monomorphic ventricular tachycardia, bigeminy and atrial fibrillation (Figure 2). Advice was sought from the UK
National Poisons Information Service, following which three hundred milligrams of amiodarone (Hameln pharma, Hameln, Germany) and three grams of magnesium (Martindale pharma, Essex, UK) was administrated via a large-bore cannula.

He was intubated following rapid sequence intubation (RSI) with ketamine (1 milligrams/kg) (Hameln pharma, Hameln, Germany), alfentanil (10 micrograms $/ \mathrm{kg}$ ) (Panpharma, Luitre, France) and rocuronium (Hameln pharma, Hameln, Germany) (1 milligrams $/ \mathrm{kg}$ ) to maintain cardiovascular stability.

Bilevel Positive Airway Pressure (BIPAP) ventilation ensured lung-protective ventilation with a tidal volume not exceeding $8 \mathrm{ml} / \mathrm{kg}$ and maximum inspiratory pressures below $30 \mathrm{cmH} 2 \mathrm{O}$. Acid-base balance identified a marginal low $\mathrm{pH}$ with raised lactate $\left(4.8 \mathrm{mmol} \cdot \mathrm{L}^{-1}\right)$ and negative base $\left(-6.9 \mathrm{mmol} \cdot \mathrm{L}^{-1}\right)$ (Table 1$)$. Cardiovascular stability was achieved, peripheral and central lines inserted before transfer (Figure 3).

Four hours following admission, assessment and stabilisation in the emergency department, he was transferred to the intensive care unit (ICU). He remained

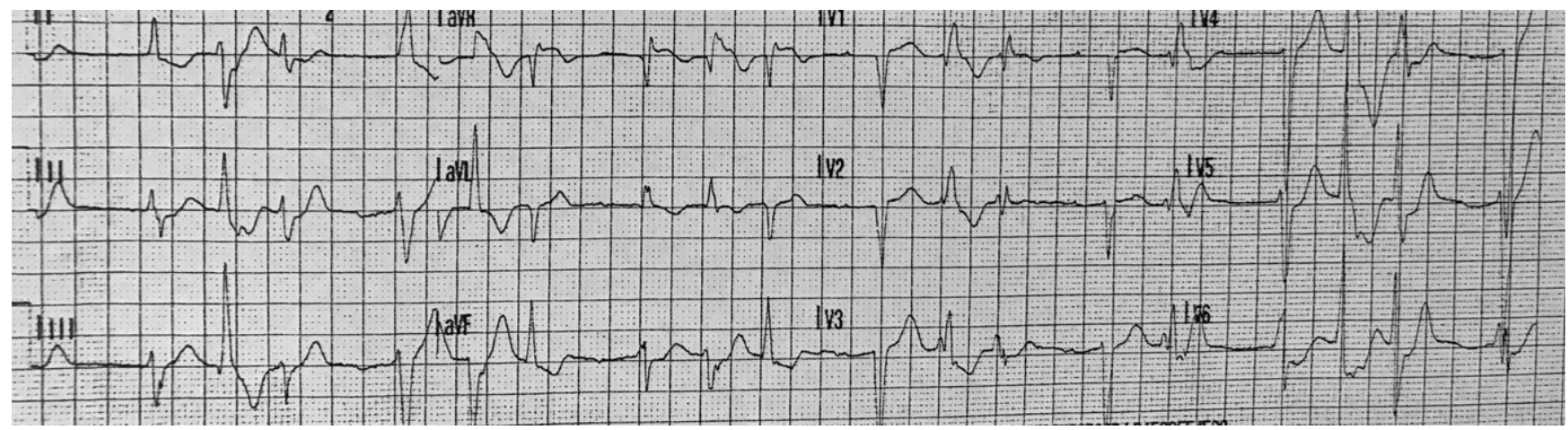

Fig. 1. 12 lead ECG, Obtained by paramedics at the pickup location

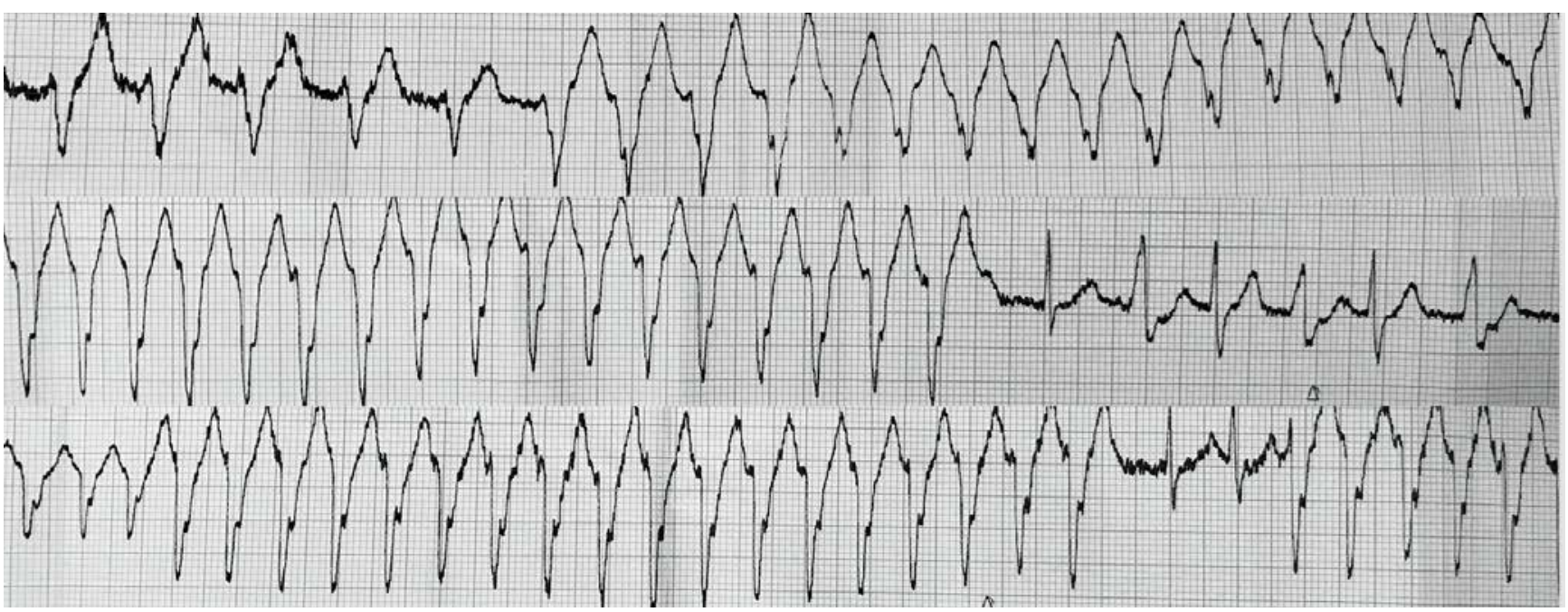

Fig. 2. Rhythm strips, obtained in the emergency department, five minutes post-admission. 
Table 1. Arterial Blood Gas (ABG) analysis following intubation. Five minutes and four hours following admission to hospital.

\begin{tabular}{|c|c|c|c|}
\hline Blood gas analysis & Intubation (FIO2 =1) & ICU (+4 hours) (FIO2 =0.35) & \\
\hline $\mathrm{pH}$ & 7.316 & 7.35 & \\
\hline $\mathrm{PaCO} 2$ & 5.03 & 5.36 & $\mathrm{kPa}$ \\
\hline $\mathrm{PaO} 2$ & 47.4 & 19.4 & $\mathrm{kPa}$ \\
\hline thb & 127 & 125 & $\mathrm{~g} / \mathrm{L}$ \\
\hline sO2 & 100 & 99 & $\%$ \\
\hline \multicolumn{4}{|l|}{ Electrolytes } \\
\hline K & 3.3 & 4.5 & $\mathrm{mmol} / \mathrm{L}$ \\
\hline $\mathrm{Ca}$ & 1.07 & 1.07 & $\mathrm{mmol} / \mathrm{L}$ \\
\hline $\mathrm{Na}$ & 141 & 142 & $\mathrm{mmol} / \mathrm{L}$ \\
\hline $\mathrm{HCO}--$ & 19.3 & 21 & $\mathrm{mmol} / \mathrm{L}$ \\
\hline \multicolumn{4}{|l|}{ Metabolites } \\
\hline Glucose & 5.5 & 6.5 & $\mathrm{mmol} / \mathrm{L}$ \\
\hline Lactate & 4.8 & 2.4 & $\mathrm{mmol} / \mathrm{L}$ \\
\hline \multicolumn{4}{|l|}{ Oxygen status } \\
\hline xBase(Ecf)c & -6.9 & -2 & $\mathrm{mmol} / \mathrm{L}$ \\
\hline
\end{tabular}

sedated with ongoing cardiovascular support via exogenous catecholamines for resistant hypotension via central venous access; noradrenaline (6 micrograms. $\mathrm{kg}-1 . \mathrm{min}^{-1}$ ) (Augettant, Lyon, France), in addition to fluid (125 ml.min ${ }^{-1}$ Ringers Lactate) and amiodarone (6 micrograms.kg-1.min $\left.{ }^{-1}\right)($ Hameln pharma, Hameln,
Germany). Acid-base balance normalised within four hours of being admitted to the ICU (Table 1).

Plasma troponin levels were not requested. Both hepatic and renal function were normal. Laboratory analysis of serum electrolyte remained within the normal range for potassium $(3.7 \mathrm{mmol} / \mathrm{l})$, magnesium

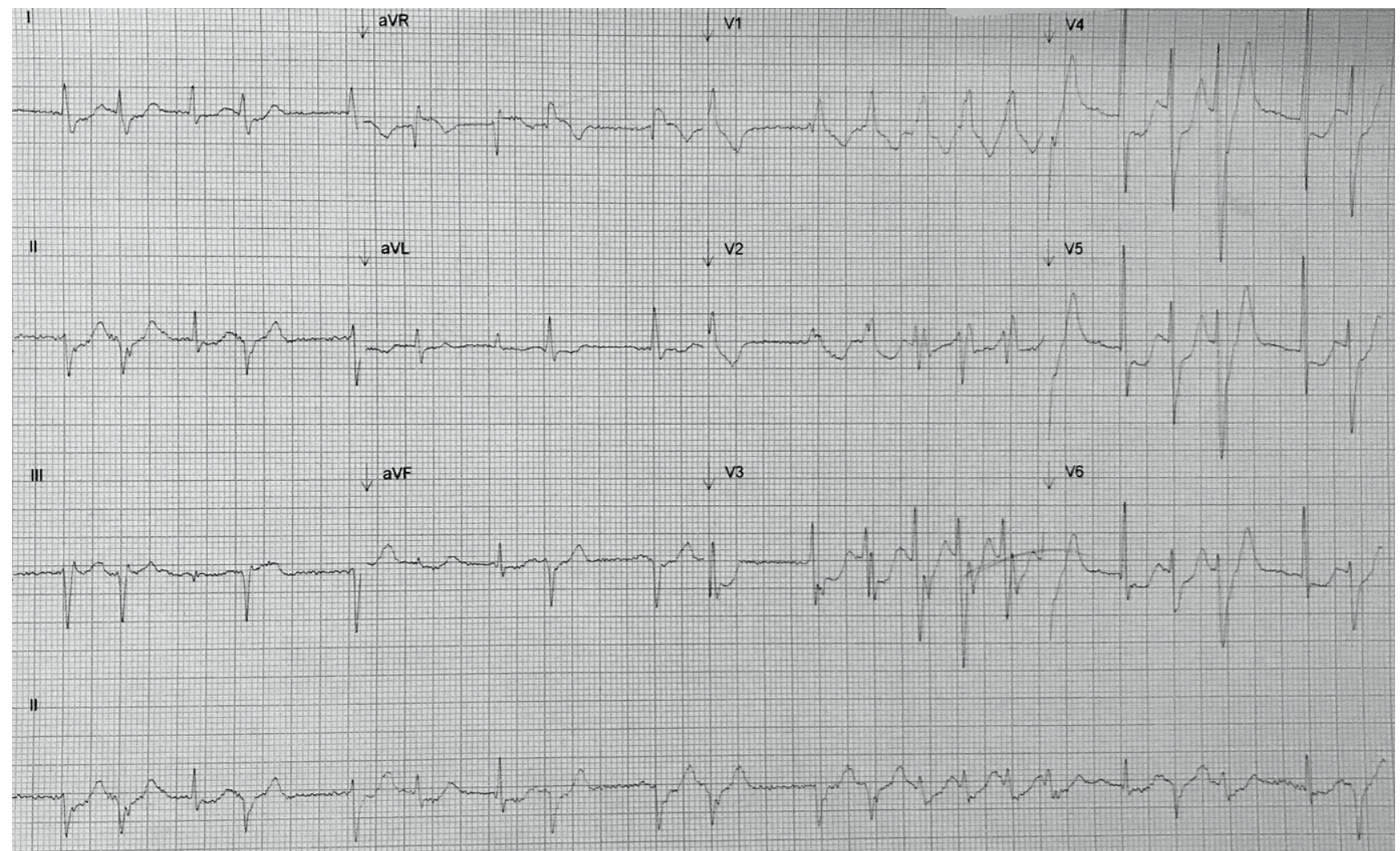

Fig 3. 12 lead ECG post amiodarone infusion, obtained in the emergency department. Two hours following admission to hospital. 
(0.77 mmol/l), sodium (142 mmol/l) and calcium (2.22 $\mathrm{mmol} / \mathrm{l})$. Unfortunately, aconite plasma concentrations were requested, but not analysed.

On day two post-admission to ICU, he remained intubated and ventilated with BIPAP ventilation. Hemodynamic stability (BP 120/65 mmHg, 67 beat.min ${ }^{-1}$ ) was achieved, and the weaning doses of noradrenaline and amiodarone were stopped (Figure 3). Three episodes of severe bradycardia were noted during early evening lasting less than one minute, all resolved spontaneously without intervention. An ECG confirmed sinus rhythm (Figure 4). Laboratory investigations remained normal.

On day three post-admission to ICU, he was successfully extubated to a $40 \%$ venture mask; he had an $\mathrm{SaO} 2$ of $99 \%$ and a respiratory rate of 18 breaths. $\mathrm{min}^{-1}$. Respiratory and cardiovascular parameters remained stable in normal sinus rhythm. Episodes of acute agitation with tachypnoea were documented during the evening lasting three hours, which were managed with intravenous midazolam (Hameln pharma, Hameln, Germany). During the early afternoon, he was reviewed by the intensive care consultant as no further level two or three care was required. He was stepped down to an acute medical ward and reviewed by psychiatric services.

\section{DISCUSSION}

Aconite is a plant indigenous through northern Asia and Europe. It is commonly grown in gardens as it has attractive purple flowers. These appear similar to the hoods worn by medieval monks, hence one of its common names, monkshood. Its potent poisonous nature has long been recognised, reputedly used by shepherds as a meat poison used to kill wolves, and by association protect against werewolves, hence the name Wolfsbane. The name aconite originates from akontion, meaning dart in Ancient Greek as arrows were dipped in aconite for their poisonous effect.

Aconite poisoning remains a rare happening in the western world, and few case studies exist for individually managed cases within the United Kingdom. Notably, in 2009, a 39-year-old man was murdered by his partner, who laced his curry with aconite, resulting in fatal consequences, leading to widespread media reporting [8]. More recently, Jacobs et al. (2018), reported a case involving a 46-year-old man who required HDU admission for single organ support following cardiovascular collapse [9].

Dedicated supportive treatment in critical care, particularly of cardiac arrhythmias, has improved outcomes and decreased mortality rates to less than six per

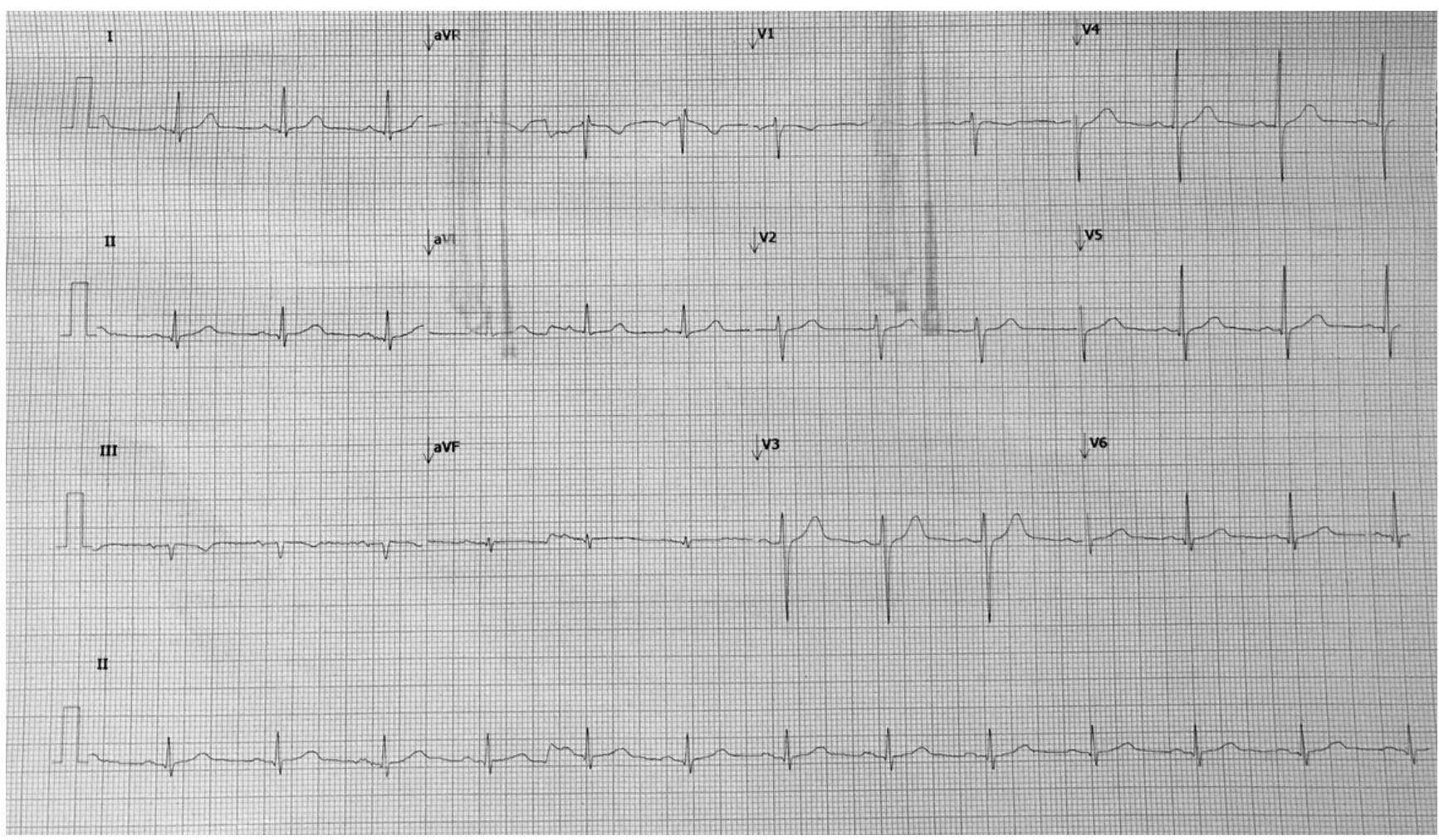

Fig. 4. 12 lead ECG 48 hours. Day two following admission. 
cent [10]. Cardiovascular manifestations are broadly classified into central effects via direct effects modulation within the ventromedial nucleus of the hypothalamus resulting in episodes of bradycardia and hypotension; conversely; agonism occurs also at peripheral sites leading to direct myocardial effects [10]. Agonist binding occurs throughout the myocardial with the site of action relating to clinical presentation, receptor binding within the sinoatrial node (SAN) has been shown to delay action potential propagation. With regards to cellular biology, recent studies have highlighted a range of cellular pathways which are modulated by aconite. These include alcium homeostasis, direct neurotoxic apoptosis, fatty acid peroxidation and cholinergic neurotransmission [11].

Aconite exerts a range of calcium-related physiological changes. These include both enhanced regulatory proteins transcription of L-type calcium channels and more specifically, $\mathrm{Na}^{+} / \mathrm{Ca}^{2+}$ bidirectional exchanger (NCX), sarcoplasmic reticulum $\mathrm{Ca}^{2+} \mathrm{ATPase}$ (SERCA2a), and directly enhancing NCX protein activity. Overall, there is an increase in both intracellular $\left[\mathrm{Ca}^{2+}\right]$ and $\left[\mathrm{Na}^{+}\right]$prolonging myocardial action potential duration, inducing triggered activities and delayed after-depolarisations [12]. A recent review article highlights not only the management and prevalence of cardiac manifestations but also the importance of voltagegated potassium channels in myocytes [12].

Direct toxic effects mediated by aconite are widespread. Peng et al. [13]. reported on direct cytotoxic effects via the inhibition of $\mathrm{Na}^{+} / \mathrm{K}^{+} /$ATPase pump, thus mediating cellular apoptosis. Conversely, rodent cell-based models report a direct correlation between aconite exposure and elevations in intracellular $\mathrm{Ca}^{2+}$ which mediate apoptotic pathways [14]. Moreover, Lin et al. 2016) reported on the direct injury observed from aconite on myocardial tissue which can mimic symptoms of acute myocardial infarction and thus stimulate the release of $\operatorname{TnT}$ [15].

Electrocardiographic manifestations following aconite poisoning have been documented in both case and review articles [12]. Our case study presents all such features, supraventricular abnormalities which include low amplitude $\mathrm{P}$-waves which are intermittently lost with episodes of sinus bradycardia. Ventricular pathologies include bundle branch block (BBB), T-wave repolarisation abnormalities, QT prolongation, sustained ventricular tachycardia, ventricular ectopy and finally, the rare bidirectional ventricular tachycardia. During this case excretion of aconite and its related alkaloids lead to the reappearance of normal P-wave morphology and atrioventricular conduction activity. Aconites $\mathrm{MAO}$ on myocardial tissue underpins these findings.

Jacobs et al. (2019) reported on a similar presentation with regards to acute collapse and severe cardiovascular instability; however, they implemented haemodialysis for toxin clearance [9]. Supportive management is the mainstay of treatment with no dedicated antidote. This includes vigilant monitoring of vital signs, with supportive cardiovascular management in the form of intravenous fluid with rhythm control involving both amiodarone for tachyarrhythmias and atropine for bradycardia. The spectrum of disease presentation is diverse with many other herbal preparations presenting similarly [16].

The clinical presentation of aconite poisoning mirrors that of other poisons. Common differential diagnoses include cardiac glycosides, andromedotoxin and shellfish poisoning. The highest mortality rates have been associated with refractory ventricular tachycardia. Treatment with amiodarone is the recommended firstline treatment followed by lidocaine and phenytoin. Case reports have reported decreasing effectiveness of reverting refractory ventricular tachycardia following failed first-line treatment intervention [9]. Prolonged cardiopulmonary resuscitation during severe intoxication with cardiopulmonary bypass has been successful [12].

Neurological manifestations relate to both central and peripheral blockade [17]. Classically, activation of voltage-gated sodium channels results in depolarization and thus reduced nerve prorogation [4]. In vitro, cell-based studies by Ameri et al. [18] have reported on extensive central based inhibition of neuronal and anti-epileptiform activity. Interestingly, aconite effects are dose dependant, low concentrations enhance presynaptic acetylcholine (Ach) release, improving muscle contraction, conversely higher concentrations lead to the prolonged opening of neuronal voltage-gated sodium channels and thus reduced synaptic Ach release [19].

\section{- CONCLUSIONS}

An interesting yet rare case of intentional aconite overdose in the United Kingdom, in a patient with a diverse disease presentation, specifically cardiac dysrhythmias and neurological compromise. Early identification 
Available online at: www.jccm.ro

with rapid treatment intervention underpins disease management.

\section{REFERENCES}

1. Chan TYK, Critchley julian AJH. Usage and adverse effects of Chinese herbal medicines. Hum Exp Toxicol. 1996;15(1):1-4.

2. Zhang H, Sun S, Zhang W, Xie X, Zhu Z, Chai Y, et al. Biological activities and pharmacokinetics of aconitine, benzoylaconine, and aconine after oral administration in rats. Drug Test Anal. 2016;8(8):839-46.

3. Wu J jun, Guo Z zhen, Zhu Y feng, Huang Z jian, Gong X, Li Y huan, et al. A systematic review of pharmacokinetic studies on herbal drug Fuzi: Implications for Fuzi as personalized medicine. Phytomedicine. 2018;44(1):187-203.

4. Gao X, Hu J, Zhang X, Zuo Y, Wang Y, Zhu S. Research progress of aconitine toxicity and forensic analysis of aconitine poisoning. Forensic Sci Res. 2018;1(1):2471-1411.

5. Fujita Y, Terui K, Fujita M, Kakizaki A, Sato N, Oikawa K, et al. Five cases of aconite poisoning: Toxicokinetics of aconitines. J Anal Toxicol. 2007;31(3):132-7.

6. Terui K, Fujita Y, Takei M, Aoki H, Endo S. Relationship between serum aconitines level and clinical features of aconite poisoning. J Tradit Med. 2008;25(3):67-73.

7. Friese J, Gleitz J, Gutser UT, Heubach JF, Matthiesen T, Wilffert $B$, et al. Aconitum sp. alkaloids: The modulation of voltage-dependent $\mathrm{Na}+$ channels, toxicity and antinociceptive properties. Eur J Pharmacol. 1997;337(2-3):165-74.

8. Bonnici K, Stanworth D, Simmonds MS, Mukherjee E, Ferner RE. Flowers of evil. Lancet. 2010;376(9752):1616.
The Journal of Critical Care Medicine 2020;6(2) • 129

9. Jacobs CO, Haydock S. ECG changes in acute aconite poisoning. QJM. 2019;112(3):227.

10. Chan TYK. Aconite poisoning. Clin Toxicol. 2009;47(4):279-85.

11. Sheikh-Zade YR, Cherednik IL, Galenko-Yaroshevskii PA. Peculiarities of cardiotropic effect of aconitine. Bull Exp Biol Med. 2000;129(4):365-6.

12. Coulson JM, Caparrotta TM, Thompson JP. The management of ventricular dysrhythmia in aconite poisoning. Clin Toxicol. 2017;55(5):313-21.

13. Peng C, Wang L, Wang YH, Li YX, Pan Y. The toxicity of aconitine, emodin on ICC cell and the anagonist effect of the compatibility. Eur J Drug Metab Pharmacokinet. 2009;34(3):213-20.

14. Liu F, Tan X, Han X, Li X, Li N, Kang W. Cytotoxicity of Aconitum alkaloid and its interaction with calf thymus DNA by multispectroscopic techniques. Sci Rep. 2017;7(1):14509.

15. Li H, Liu L, Zhu S, Liu Q. Case reports of aconite poisoning in mainland China from 2004 to 2015: A retrospective analysis. J Forensic Leg Med. 2016;42(1):68-73.

16. Yilmaz O, Eser M, Sahiner A, Altintop L, Yesildag O. Hypotension, bradycardia and syncope caused by honey poisoning. Resuscitation. 2006;68(3):405-408.

17. Ameri A. Electrophysiological actions of the plant alkaloid 6-benzoylheteratisine in rat hippocampal slices. Naunyn Schmiedebergs Arch Pharmacol. 1997;355(4):538-44.

18. Ameri A. The effects of Aconitum alkaloids on the central nervous system. Prog Neurobiol. 1998;56(2):211-35.

19. Yeih DF, Chiang FT, Huang SK. Successful treatment of aconitine induced life threatening ventricular tachyarrhythmia with amiodarone. Heart. 2000;84(4):8. 